\title{
PLANT LEAF RECOGNITION SYSTEM USING KERNEL ENSEMBLE APPROACH
}

\author{
Dr. Phiros Mansur, \\ Department of CNET, \\ College of Computer Science and Information, \\ Jazan University, KSA \\ firosmansur@jazanu.edu.sa
}

\begin{abstract}
The information about the classification of plant leaf into appropriate taxonomies is very useful for botanists. In this study, an efficient Plant Leaf Recognition (PLR) system is designed using kernel ensemble approach by Support Vector Machine (SVM). At first, the plant leaf images are normalized and resized by color normalization and bicubic interpolation. Features such as $4^{\text {th }}$ order color moments and nine energy maps of LAWS are combined to form a feature database. The classification is done by ensemble approach with different SVM kernels like Linear (SVM-L), Radial basis function (SVM-R), Polynomial (SVM-P) and Quadratic (SVM-Q). Finally, the outputs of each SVM classifier are fused to classify plant leaf images. The PLR system is carried on using Folio database that contains 640 leaf images captured from 32 species. The system achieves $90.63 \%$ recognition rate by the ensemble approach using colour moments and texture features by LAWS.
\end{abstract}

Keywords: Plant leaf recognition, colour moments, LAWS texture, ensemble classification

\section{INTRODUCTION}

Now-a-days, computer vision methodologies are used to recognize plants automatically. A deep learning approach for PLR system is discussed in [1]. It uses convolution neural network with softmax for the classification. To speed up the process, cropping is employed before extracting features and classification. The networking depth is adjusted by GoogleNet. Shape features for PLR is described in [2] using neural network. The moment invariant features are used as shape features to classify three plants. Multi Layer Perceptron (MLP) is used as classifier.

Geometric features along with twelve morphological features are used in [3] to classify 32 species. Probabilistic neural network is used to recognize the leaf. Also, principal component analysis is employed as a dimensionality reduction approach. A competitive based algorithm is used for PLR in [4]. To build the model, histogram colour data and set of shape data are used. Three different classifiers such as random forest, Support Vector Machine (SVM) and learning vector quantization are used to recognize leaf images.

An approach for PLR using leaf's venation and shape is discussed in [5]. Different features such as edges by canny, moment $\mathrm{s}$, centroid-radii model and leaf veins are extracted. The prediction is done by MLP architecture. Leaf contour based features is described in [6] for PLR. A histogram is formed using distances from the center of the leaf to the contour. Bayes theorem is used for the classification of thirteen species.

A PLR system using histogram of oriented gradients and Zernike moments is discussed in [7]. The petioles are removed using distance transform and the image is aligned to center before extracting features. SVM classifier is used and the features are normalized before classification. A multi-scale distance matrix 
based PLR system is described in [8]. It captures the shape geometry using Euclidean distance. Then, Decomposed Newton's Method is employed to reduce the dimensionality along with Maximum Margin Criterion.

Gabor features with low dimensional embedding based PLKR system is discussed in [9]. AT first, petioles are removed by morphological operation and then converted into gray scale after resized. Gabor features are extracted at eight directions. K-nearest neighbor classifier is used. A layered approach based PLR system is described in [10]. Each layer uses different features such as colour, texture and shape to create a model. A neuro fuzzy classifier is employed to recognize the leaf images.

In this study, an efficient PLR system is designed with the help of colour moments and LAWS texture features. The organization of the paper is as follows: Section 2 gives the overview of the extracted features and classifiers used in the PLR system. In section 3, the obtained results of PLR system are discussed and conclusions derived from the performance of PLR system is given in the last section.

\section{METHODS AND MATERIALS}

The PLR system consists of three stages. The first stage is pre-processing which removes illumination variations of captured leaf images. A colour normalization technique is used to remove illumination variation and bi-cubic interpolation is used to resize the image into a fixed resolution. The color components (Red, Blue and Green) are separated for feature extraction after preprocessing. In feature extraction, up to $4^{\text {th }}$ order color moments are extracted from red, green, and blue components at first. Then, a set of nine $5 \times 5$ masks is used to extract the LAWS texture feature from red, green and blue components independently. Finally the feature database is used to train SVM classifier with its kernels RBF, linear, quadratic and polynomial and hybridized to classify the plant leaf.

\section{A. Preprocessing}

In this stage, normalization and rescaling are employed to improve the performance of PLR system. At first, simple colour normalization is utilized to remove the illumination assume that the imaging camera has linear camera response. Thus, the pixel intensities in each colour channels are modified by (1)

$$
(r, g, b)=(n r, n g, n b) \text { where } n=\frac{1}{r+g+b}
$$

where $n$ indicates normalization factor. After normalization, the image is rescaled to a standard size by bi-cubic interpolation approach [11].

\section{B. Creation of Feature Database}

Figure 1 shows the feature extraction of PLR system. Texture is an important feature in many computer vision applications. To measure the variation within a specified fixed size window, Laws [12] described texture energy approach which uses a set of nine 2D-5x5 masks obtained from four $1 \mathrm{D}-1 \mathrm{x} 5$ vectors that detects ripples, edges, spots and levels. The $1 \mathrm{D}-1 \mathrm{x} 5$ vectors are as follows: 


$\begin{array}{llllllllll}\text { R5 } & \text { (Ripple) } & = & {[} & 1 & -4 & 6 & -4 & 1 & ] \\ \text { L5 } & (\text { Level }) & = & {[} & 1 & 4 & 6 & 4 & 1 & ] \\ \text { S5 } & (\text { Spot }) & = & {[} & -1 & 0 & 2 & 0 & -1 & ] \\ \text { E5 } & (\text { Edge }) & = & {[} & -1 & -2 & 0 & 2 & 1 & ]\end{array}$

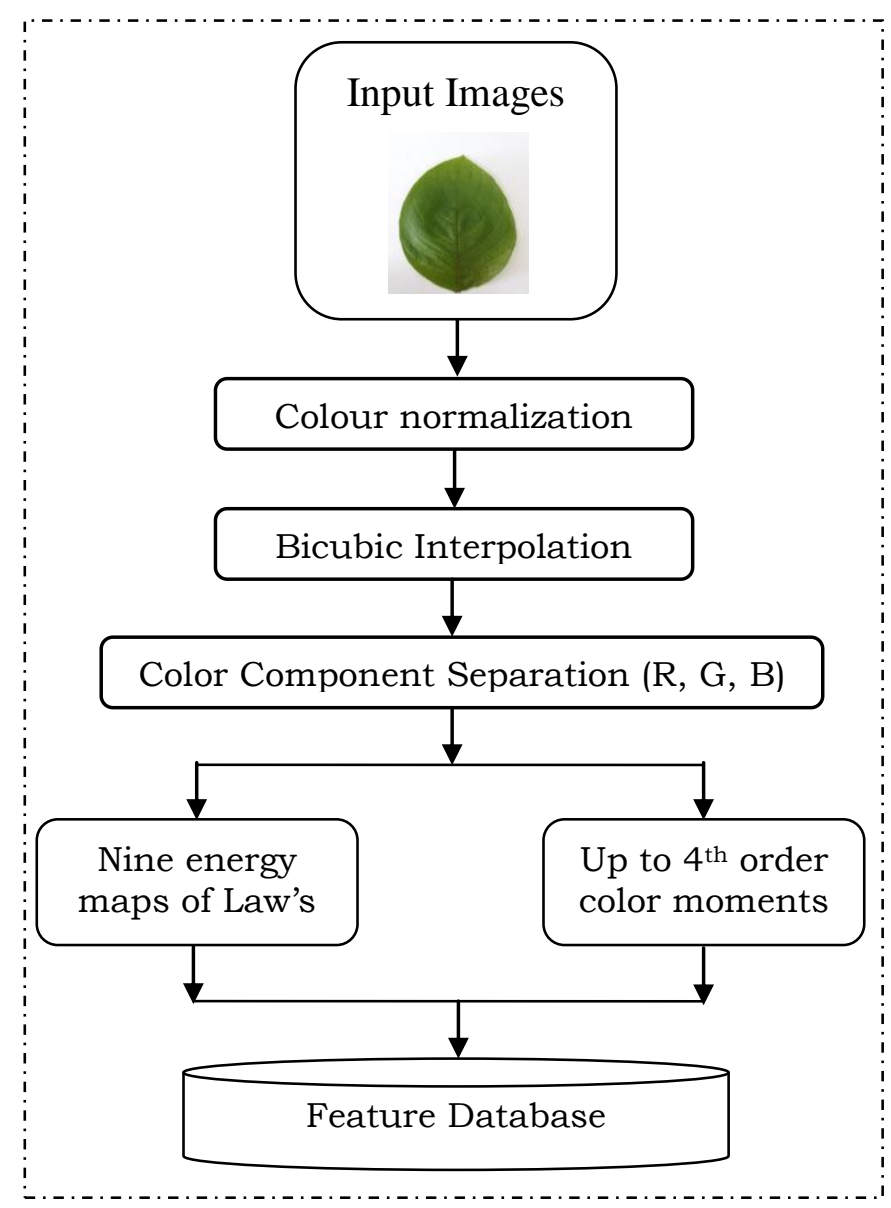

Fig. 1 Feature extraction of PLR system

From the 1D vector, sixteen 2D masks are obtained by convolving two 1D vectors. Among them, the symmetric pairs are removed and finally, nine 2D masks are computed. These masks are convolved with the plant leaf images to produce texture features. Also, the colour features are computed up to $4^{\text {th }}$ order moments such as mean, standard deviation, skewness and kurtosis from each colour channels.

\section{Ensemble Classification}

The recognition of plant leaf is made by the SVM classifier with kernel ensemble classification. The SVM classifier with different kernels such as SVM-L, 
SVM-R, SVM-P, and SVM-Q are trained with different samples. The kernel functions are defined as $k\left(x_{i}, x_{j}\right)=\phi\left(x_{i}\right) \phi\left(x_{j}\right)$. Here the vector $x$ is mapped from the space $\phi(x)$ to some other Euclidean space. The outputs of these SVM classifiers are analyzed to take final decision for the recognition. The kernel functions are as follows:

$$
\begin{gathered}
k(x, y)=x^{T} \cdot y+c \\
k(x, y)=\left(\alpha x^{T} \cdot y+c\right)^{d} \\
k(x, y)=\exp \left(\frac{\|x-y\|^{2}}{2 \sigma^{2}}\right) \\
k(x, y)=1-\frac{\|x-y\|^{2}}{\|x-y\|^{2}+C}
\end{gathered}
$$

Figure 3 shows the overall classification stage for SVM classifier with different kernels.

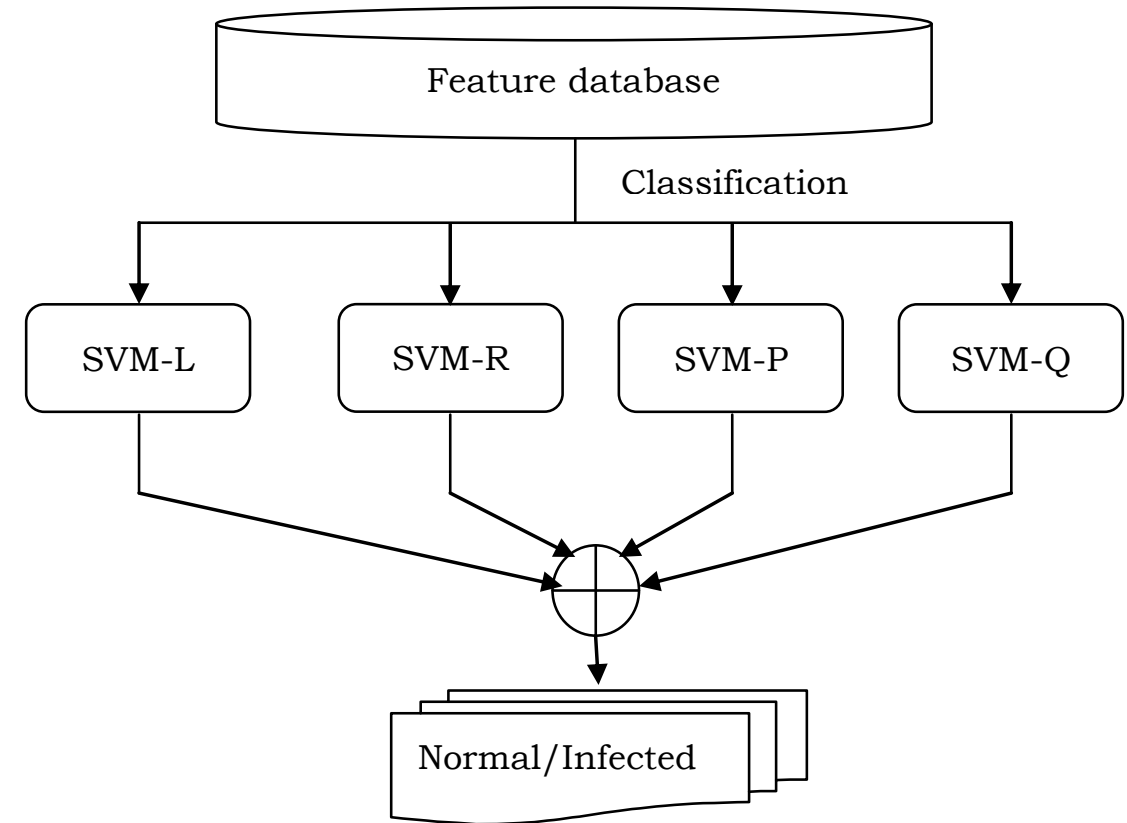

Fig. 2 Ensemble Approach of PLR system

\section{RESULTS AND DISCUSSION}

The performance of PLR system is validated using Folio [13-14] database. The leaf images in the database are acquired from Mauritius. It consists of 640 leafs of 32 different species (20 images/species). The resolution of all leaf images is $1980 \times 1024$ pixels. In order to increase the speed of the PLR system, all images are resized to $256 \times 256$ pixels in the preprocessing stage. Figure 3 shows the images from the Folio database. 

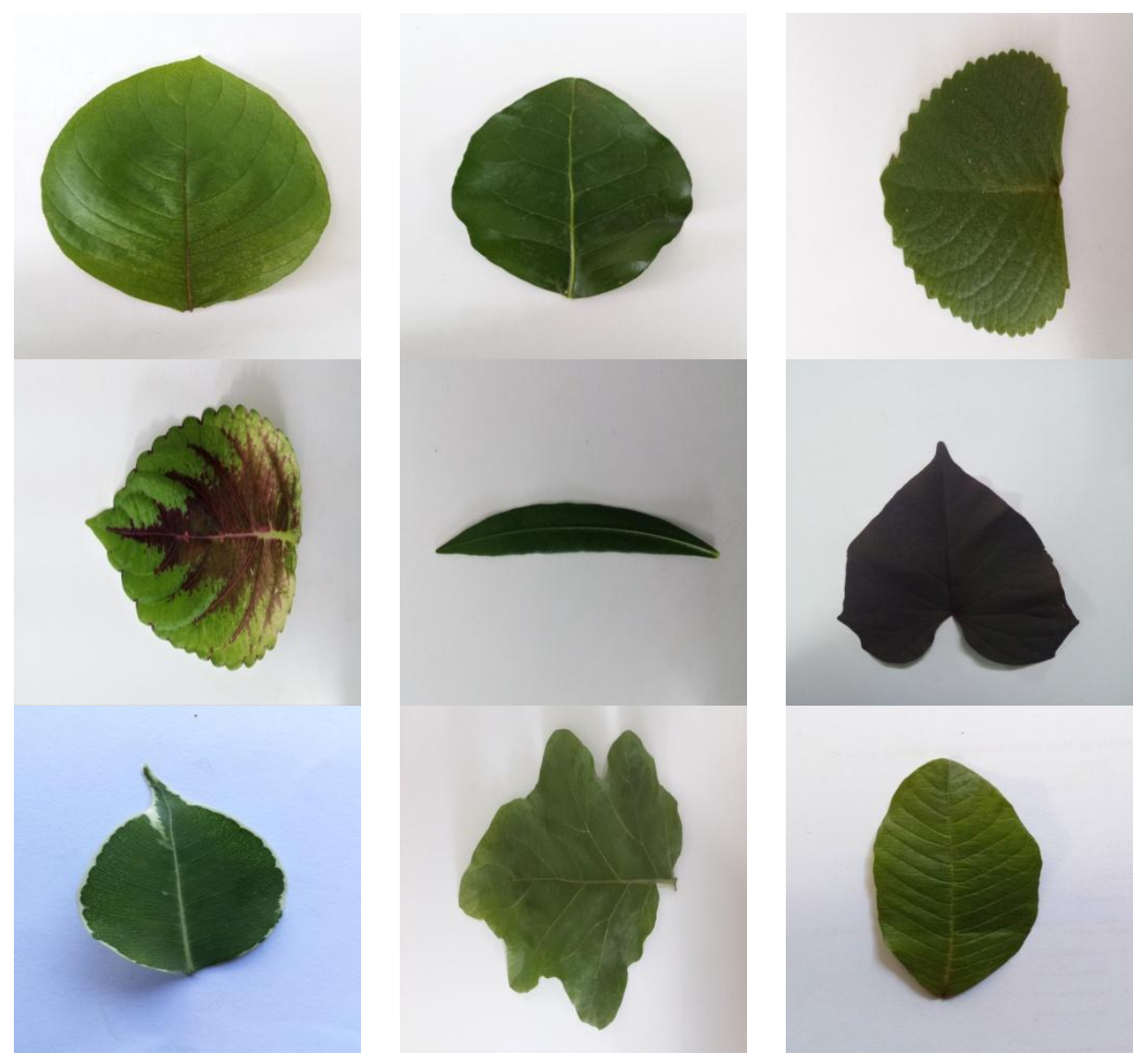

Fig. 3 Folio database plant leaf images

The performance of PLR system is measured in terms of accuracy. The system classifies the plant leaf by ensemble approach with four different kernels in SVM. Thus, the dataset is divided randomly into two sets with $60 \%$ images for training and $40 \%$ for testing. This process is repeated 4 times so that each classifier is trained with different training samples and tested with different testing samples. Finally, the ensemble approach uses their individual performance to make final decision. Table 1 shows the performance of PLR system in terms of accuracy.

TABLE 1 Performance of PLR system

\begin{tabular}{|c|c|c|c|}
\hline \multirow{2}{*}{ Classifier } & \multicolumn{3}{|c|}{ Accuracy } \\
\cline { 2 - 4 } & Colour Moments & $\begin{array}{c}\text { LAWS Texture } \\
\text { Features }\end{array}$ & $\begin{array}{c}\text { Colour Moments + } \\
\text { LAWS Features }\end{array}$ \\
\hline SVM-L & $70.31 \%$ & $75.00 \%$ & $79.69 \%$ \\
\hline SVM-R & $74.61 \%$ & $79.69 \%$ & $87.89 \%$ \\
\hline SVM-Q & $69.53 \%$ & $74.22 \%$ & $76.17 \%$ \\
\hline SVM-P & $69.14 \%$ & $73.44 \%$ & $75.39 \%$ \\
\hline Ensemble & $79.69 \%$ & $84.38 \%$ & $90.63 \%$ \\
\hline
\end{tabular}


The performance of PLR system gives 90.63\% accuracy while using ensemble classifier with the combined features. The combined features provide $6 \%$ accuracy over then LAWS features and $10 \%$ than colour moments. When compared to each classifier, the SVM-R gives promising results of $87.89 \%$ for combined features than others. It is noted that there is no comparable difference between the performance of SVM-Q and SVM-P.

\section{CONCLUSION}

An efficient PLR system based on moments and texture features is presented. The colour information in the leaf image is processed independently to achieve higher recognition rate. Before feature extraction, the input leaf image is normalized and rescaled using colour normalization and interpolation techniques. A combined feature set with colour moments and LAWS texture map is used fort effective recognition by ensemble approach. The performance of PLR system is analyzed using Folio database images with ensemble approach and also with individual classifier. Also, the effect of extracted features is analyzed independently by the classifier. Results show that the ensemble approach with combined features provides better recognition rate than their individual counterpart.

\section{REFERENCES}

[1]. W.S. Jeon, and S.Y. Rhee, "Plant leaf recognition using a convolution neural network", International Journal of Fuzzy Logic and Intelligent Systems, Vol. 17, No. 1, 2017, pp. 26-34.

[2]. J. Chaki, and R. Parekh, "Plant leaf recognition using shape based features and neural network classifiers", International Journal of Advanced Computer Science and Applications, Vol. 2, No. 10, 2011, pp. 41-47.

[3]. S.G. Wu, F.S. Bao, E.Y. Xu, Y.X. Wang, Y.F. Chang, and Q.L. Xiang, "A leaf recognition algorithm for plant classification using probabilistic neural network", International symposium on signal processing and information technology, 2007, pp. 11-16.

[4]. E.M. Imah, Y.S. Rahayu, and A. Wintarti, "Plant leaf recognition using competitive based learning algorithm", IOP Conference Series: Materials Science and Engineering, Vol. 288, No. 1, 2018, pp. 012058.

[5]. A.L. Codizar, and G. Solano, "Plant leaf recognition by venation and shape using artificial neural networks", International Conference on Information, Intelligence, Systems \& Applications, 2016, pp. 1-4.

[6]. C.Y. Gwo, and C.H. Wei, "Plant identification through images: Using feature extraction of key points on leaf contours1", Applications in plant sciences, Vol. 1, No. 11, 2013, pp. 1-9.

[7]. D.G. Tsolakidis, D.I. Kosmopoulos, and G. Papadourakis, "Plant leaf recognition using Zernike moments and histogram of oriented gradients", In Hellenic Conference on Artificial Intelligence, 2014, pp. 406-417.

[8]. R. Hu, W. Jia, H. Ling, and D. Huang, "Multiscale distance matrix for fast plant leaf recognition", IEEE transactions on image processing, Vol. 21, No. 11, 2012, pp. 4667-4672.

[9]. Q. Wang, J. Zhao, M. Li, C. Cao, and Y. Lei, "Preserving discriminant manifold subspace learning for plant leaf recognition" International 
Conference on Natural Computation, Fuzzy Systems and Knowledge Discovery, 2016, pp. 1744-1749.

[10]. J. Chaki, R. Parekh, and S. Bhattacharya, "Plant leaf recognition using a layered approach", International Conference on Microelectronics, Computing and Communications, 2016, pp. 1-6.

[11]. R. Keys, "Cubic convolution interpolation for digital image processing". IEEE Transactions on Acoustics, Speech, and Signal Processing. Vol. 29, No. 6, 1981, pp. 1153-1160.

[12]. K. Laws, "Textured Image Segmentation", Ph.D. Dissertation, University of Southern California, 1980.

[13]. T. Munisami, M. Ramsurn, S. Kishnah, and S. Pudaruth, "Plant leaf recognition using shape features and colour histogram with K-nearest neighbour classifiers", Procedia Computer Science, Vol. 58, 2015, pp. 740747.

[14]. Folio Database: https://archive.ics.uci.edu/ml/datasets/Folio 